\title{
MICROSTRUCTURE AND SLIDING WEAR BEHAVIOUR OF STIRCAST Ti-Gr AND Ti-Fe METAL MATRIX COMPOSITES
}

\author{
S.S Sharma ${ }^{1}$, Gowri Shankar M.C ${ }^{2}$, U. Achutha Kini ${ }^{3}$, Shivaprakash Y.M ${ }^{4}$, P. R. Prabhu ${ }^{5}$ \\ ${ }^{1}$ Professor, Mechanical \& Manufacturing Engg. Dept, MIT, Manipal, Karnataka, India \\ ${ }^{2}$ Assistant Professor-Sr.Scale, Mechanical \& Manufacturing Engg. Dept, MIT, Manipal, Karnataka, India \\ ${ }^{3}$ Professor, Mechanical \& Manufacturing Engg. Dept, MIT, Manipal, Karnataka, India \\ ${ }^{4}$ Assistant Professor-Sr.Scale, Mechanical \& Manufacturing Engg. Dept, MIT, Manipal, Karnataka, India \\ ${ }^{5}$ Associate Professor, Mechanical \& Manufacturing Engg. Dept, MIT, Manipal, Karnataka, India
}

\begin{abstract}
The type and weight percentage of reinforcement play important role in microstructure and sliding wear resistance of the composite. The low melting point nonferrous metal matrix composites Tin-Graphite and Tin-Iron metal matrix composites are cast by the liquid stir casting technique with up to 5 and 15 weight percentages of carbon and iron respectively as reinforcements. The sliding wear characteristics are important if the specimens are used as bearing materials for light duty applications. The specimens are polished and etched for microstructure examination to verify the homogeneous dispersion of reinforcements in the matrix. The microstructure examination reveals the uniform dispersion of reinforcements in the matrix. The specimens are also subjected to dry sliding wear on Pin -on-disc wear testing equipment and the weight loss method technique was used to find specific wear rate at the respective sliding distance. The cumulative specific wear rates and friction coefficients are plotted against sliding distance. The specific wear rate curves show the necessity of an optimum weight percentage of reinforcements in the composite to reduce the wear rate. The frictional coefficient versus sliding distance curves show the decrease in frictional force at higher sliding distances as the weight percentage of particulates increases. At higher sliding distances, in case of the Tin -Graphite composite the surface of the specimen observed to be smooth which makes the graphite particles to expose as flakes and act as solid lubricant to reduce wear. The scanning electron microscope image of the surface which is normal to the sliding surface also studied to verify the flake formation during wear. Both mild and severe wear regions are observed.
\end{abstract}

Keywords: particulate, metal matrix composites, stir casting, specific wear rate, friction coefficient.

\section{INTRODUCTION}

In present decade metal matrix composites are attracting enormous interest to researchers [1,2 and 3]. In low temperature application bearings to improve wear resistance, it is compulsory to apply a lubricant to reduce wear. Self lubricated bearing composite material containing lubricant exposed automatically during the use has therefore become an important branch of wear material and requires careful research to prevent degradation and save the life of component [4 and 5]. Most of the research articles come up with a number of technical solutions for the wear and frictional problems in metal matrix composites and provided a major framework for the practical analysis [6, 7 and 8]. One of the most important solid lubricant materials used is graphite. During the optimum conditions the metal graphite composites become self lubricating by transferring graphite flakes to the contacting surface as thin film to prevent a direct physical contact between the mating surfaces $[9,10$ and 11]. The success rate of metal matrix composites depends on the ability of the graphite flakes to come out of their embedded form from the matrix and spread evenly in the form of a solid metastable lubricating film over the abrading surfaces to provide lubrication. Wear resistance and hardness increase was observed for the optimum size and quantity of Graphite particles in the matrix [12 and 13]. There is influence of different loads, abrasive particle size of wear disc and sliding distance on the wear rate while experimenting on pin-on-disc wear testing machine [14, 15 and 16]. The wear tests were performed under dry conditions with a pin-on-disc machine. There is provision to change speed of the disc, track radius and load on specimen during testing. The pin-on-disc apparatus is a sturdy versatile machine, which facilitates study of friction and wear characteristics in sliding contacts under desired conditions. The sliding occurs between the stationery pin and a rotating disc. The normal load, rotational speed and wear track diameter can be varied to suit the test conditions. The tangential frictional force in Newton and wear in micrometers are monitored with electronic sensors and recorded.

This study presents systematic investigation on the microstructure of Tin-Graphite and Tin - Iron metal matrix composites. The distribution and interparticle distance play important role in deciding the property of composites. Study also focuses on the possibility of agglomeration or poor dispersion of reinforcements in the matrix. There are limited studies on wear behaviour of low melting temperature metal matrix composites containing Graphite as the reinforcement. In the literature, previous studies were mostly concentrated on conventional alloys containing solid solutions or intermediate phases. 


\section{EXPERIMENTAL DETAILS}

\subsection{Wear Study}

The dry sliding wear pattern is studied. The experiments are conducted on pin-on-disc wear testing machine. The specimens are prepared using micro-cut machine and subsequently turned to the required size. The standard cylindrical specimen of $8 \mathrm{~mm}$ diameter and length $20 \mathrm{~mm}$ is used for the study. First the specimen is weighed and positioned on the specimen holder of the machine. The track diameter and disc speed were selected as $110 \mathrm{~mm}$ and 960rpm respectively. Each specimen was run for two hours and weight loss was noted for every 15 minutes of disc running. Before taking the actual readings a trial run of 30 minutes is provided to each specimen. The experiment was repeated for two samples and the average weight loss was considered. The specific wear rate was calculated from the equation 1 .

\section{Specific wear rate $=\frac{\text { volume loss }}{\text { sliding distnace } \mathrm{x} \text { load }} \frac{\mathrm{mm}^{3}}{\mathrm{Nm}}$} (1)

Where, Volume $=$ Weight/Density

Sliding distance in meters for 15 mins of run

$(\mathrm{m})=\pi \mathrm{D} \mathrm{N} x 15 / 1000$

Track radius $(\mathrm{D})=110 \mathrm{~mm}$.

$\mathrm{RPM}$ of $\operatorname{disc}(\mathrm{N})=960$

Load $=500$ grams

The behaviour of the composite with respect to the wear rate is analyzed by plotting the graph of cumulative specific wear rate against sliding distance. Friction coefficient values are also compared with sliding distances. To analyze the surface and subsurface of the wear region, the SEM image of the surface, which is normal to the contacting surface is analyzed.

\subsection{Microstructure Analysis}

Initially major burrs are removed using belt polisher. Further, specimens are polished with emery papers of $1 / 0$, $2 / 0,3 / 0$, and 4/0. Mirror like polished surface is obtained by polishing on disc polisher where the polishing disc is covered with velvet clothe. Fine alumina paste is used as abrasive during polishing on disc polisher. Specimens are washed with cold water and quickly dried with ethanol. Dried specimens are etched with Nital, washed and dried after exposing for optimum duration with etchant. Inverted metallurgical microscope is used to record the microstructure at $200 \mathrm{X}$.

\section{RESULTS AND DISCUSSION}

\subsection{Microstructural Features}

The microscopic images of polished and etched surfaces for different Tin-Carbon composites are shown in Figure 1 and different Tin-Iron composites are shown in Figure 2.
Microstructure of both types of composites are recorded and analyzed. In both verities there is uniform dispersion of particles in the matrix. There was no agglomeration of reinforcements since sufficient care was taken to prevent agglomeration by preheating the particulates to $300^{\circ} \mathrm{C}$. Microstructures show the uniform dispersion of Graphite particles on the matrix.

\subsection{Wear Property}

Figure 3 shows the results of wear test as specific wear rate versus sliding distance for Tin - Graphite composites. During the beginning of run the wear rate of all the Tin Graphite composites are similar and also severe. This may be due to the improper contact of the mating specimen surface with the disc surface. During the moderate running durations, one weight percentage Graphite reinforced composite shows high wear resistance as compared to other variety graphite groups. The strain hardening index of Iron is higher than graphite; the strain hardening effect reduces the wear resistance. Increase in weight percentage of graphite reduces strain hardening effect, which increases wear rate during moderate sliding distances. At higher sliding distances the wear rate decreases as the weight percentage of particulate increases in the matrix. As the weight percentage of graphite increases wear resistance decreases at higher sliding distances. During abrasion, the graphite flakes expose to the surface at higher sliding distance and the volume percentage of flakes exposed increases with the weight percentage of reinforcements present in the composites to reduce the wear. Figure 4 shows the graph of friction coefficient versus sliding distance for the Tin Graphite composites. There is decrease in the friction coefficient values as the weight percentage of Graphite increases in the composites. The synergetic effect of strain hardening and surface flake formation may be responsible to these phenomena. Figures 5 and 6 show similar trend with better wear resistance and lesser frictional coefficient values compared to Tin - Graphite composites. As the weight percentage of Iron increases in the composite, the higher strain harden index Iron particles present in the matrix strongly increases work hardening effect as the running distance increases. Hence slope of the specific wear rate versus sliding distance curve is less compared to that of Tin - Graphite curves. Figure 6 shows the average steady friction coefficient values for greater percentage iron reinforced composites even at higher sliding distance. Figure 7 shows the SEM image for flake formation on the surface and subsurface layers of the contacting surface for 5 weight percentages of Graphite in the composite. This shows the solid lubricating effect of the composite during sliding to reduce the wear. 


\section{FIGURES}

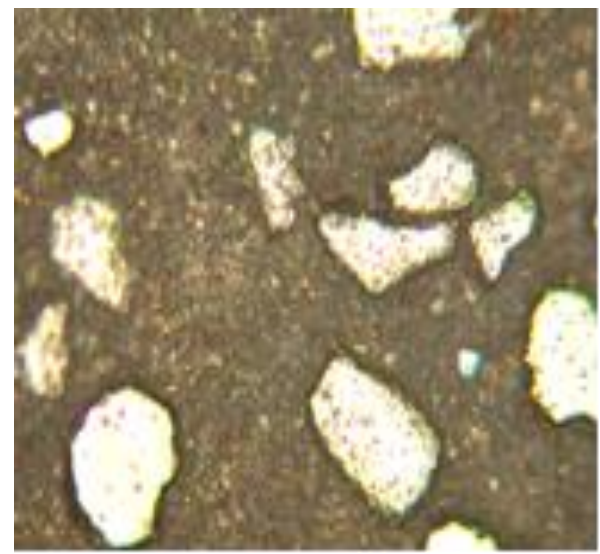

(a)

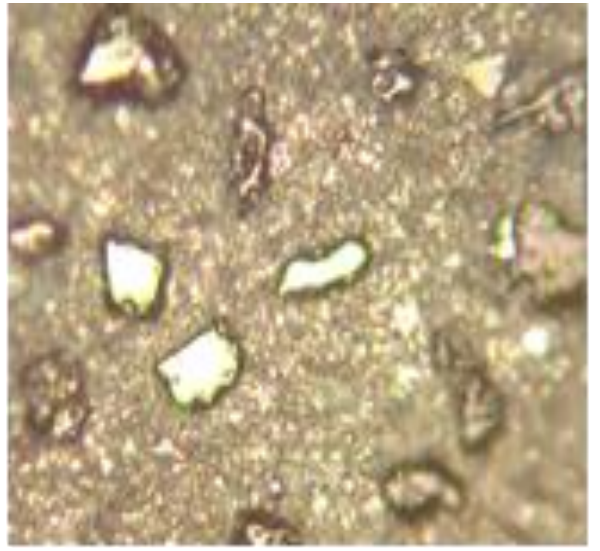

(b)

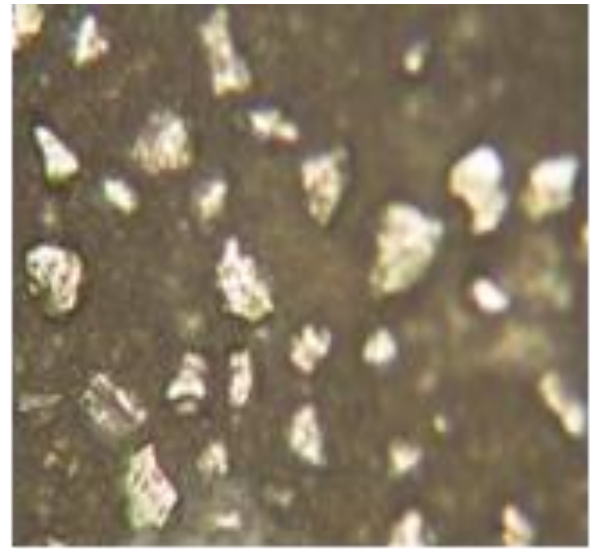

(c)

Fig 1 Microstructures of a) 1\%, b) 3\%, c) 5\% weight percentages graphite reinforced with Tin at 200x.

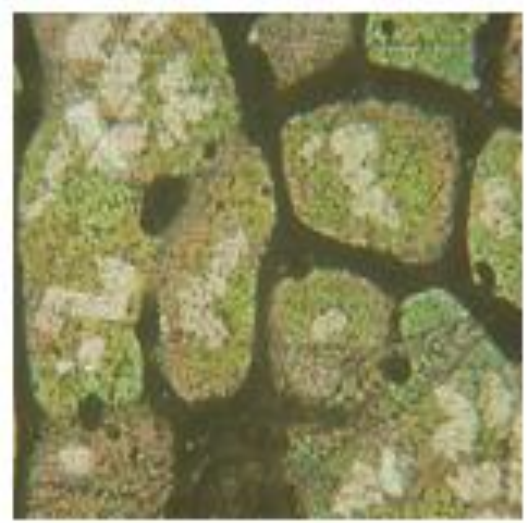

(a)

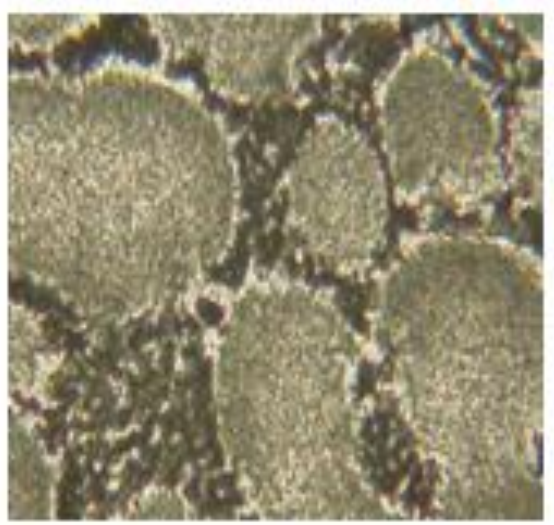

(b)

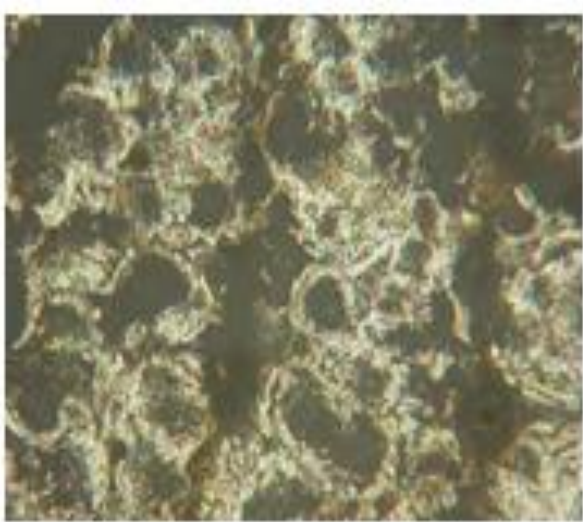

(c)

Fig 2 Microstructures of a) 5\%, b) 10\%, c) 15\% weight percentages Iron reinforced with Tin at 200x.

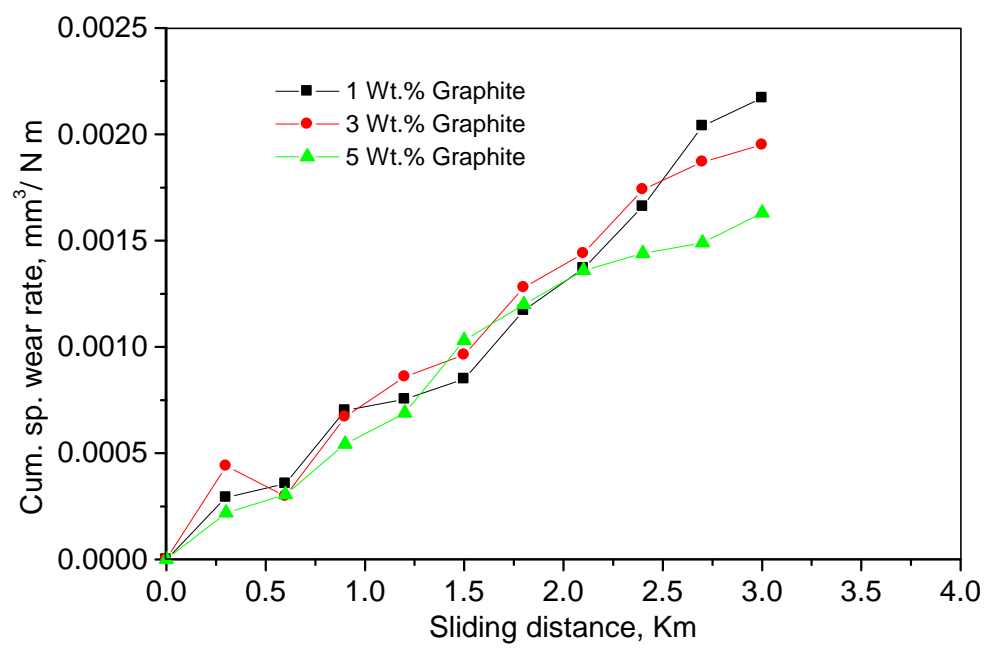

Fig 3 Wear rates for Tin-graphite composites 


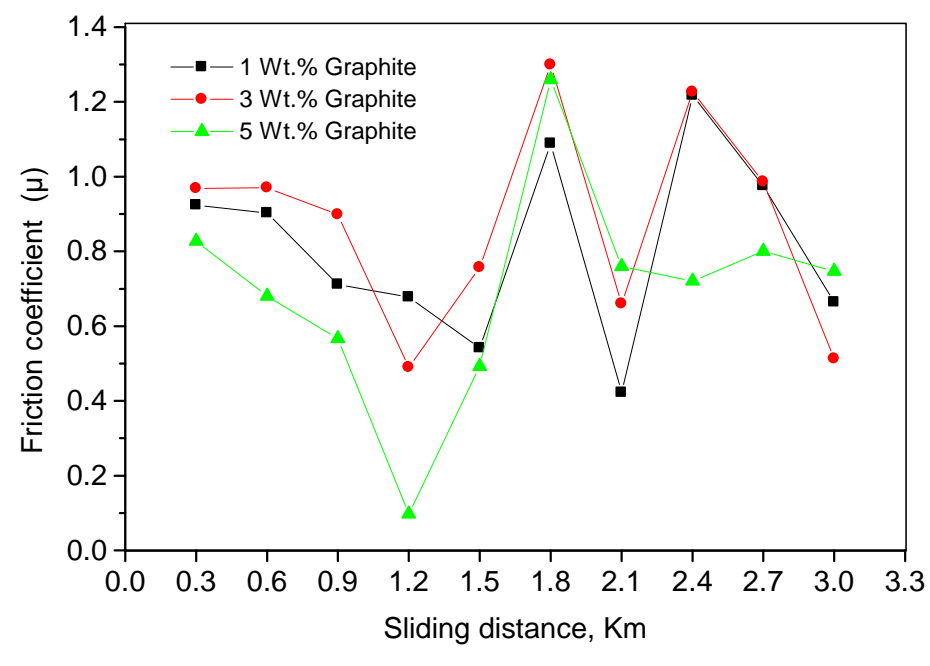

Fig 4 Friction coefficient vs. sliding distance

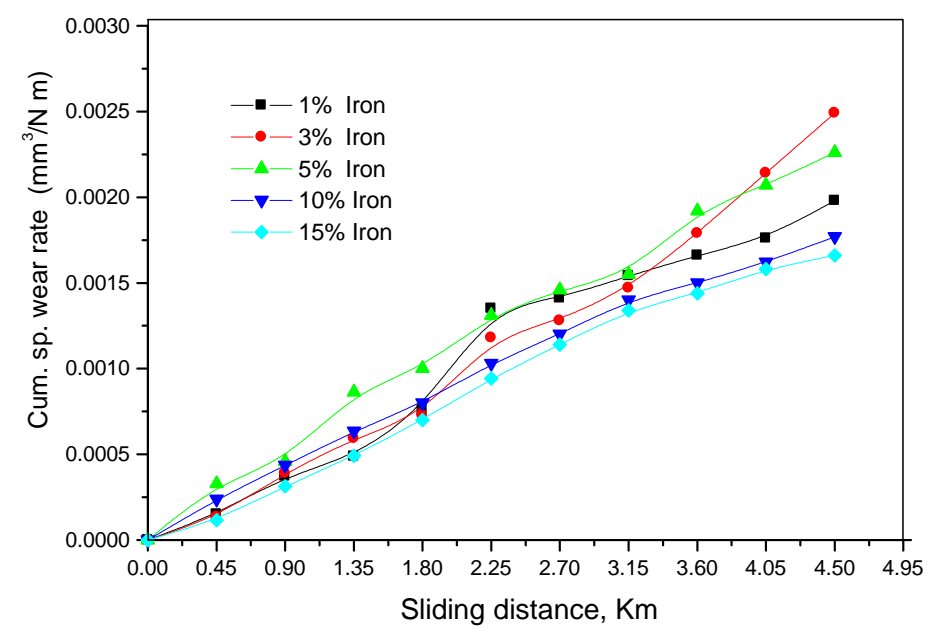

Fig 5 Wear rates for Tin-Iron

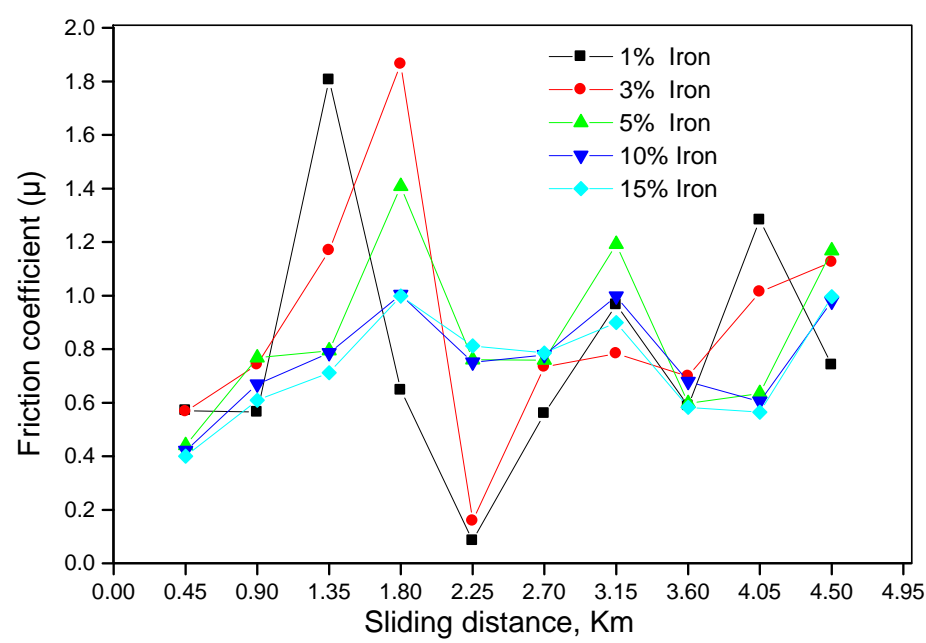

Fig 6 Friction co-efficients for Tin-Iron 

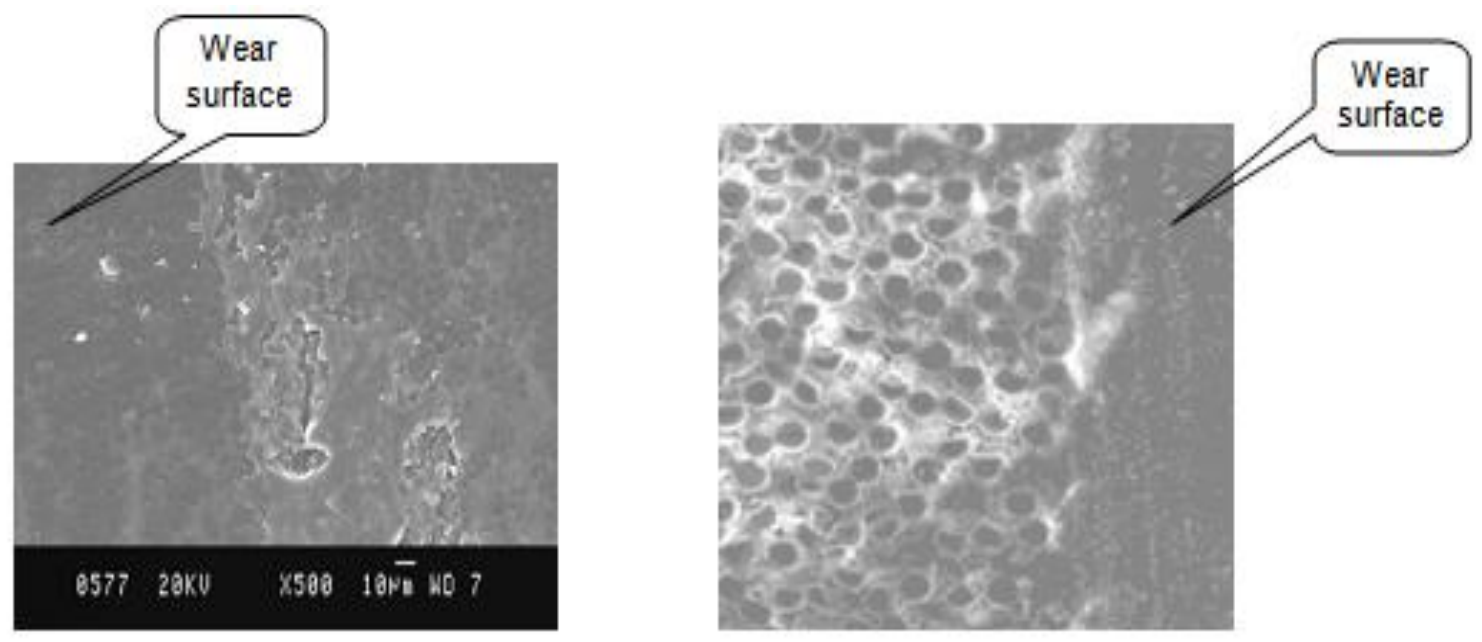

Fig 7 SEM image of Tin-Carbon composite graphite Flake formation on wear surface

\section{CONCLUSION}

Inspite of dispersion problem of reinforcement in stir casting process due to the difference in densities of matrix and reinforcement, it is possible to obtain sound casting.

However following conclusions can be drawn from the results of the present study conducted on Lead-Graphite composites.

- Even though Graphite is softer the solid lubricating effect reduces the wear rate at longer sliding distances and is evident from the SEM Image.

- Friction coefficient values of the composites decreases at higher sliding distances with the increase in the weight percentage of both types of reinforcements.

- Friction coefficient values remains almost constant at higher sliding distances for 5 weight percentage of Graphite in the composites.

- Slope of the specific wear rate versus sliding distance curve is less for Tin - Iron composites compared to that of Tin - Graphite curves.

- Wear rate and friction coefficient are minimum at higher sliding distances for the higher weight $\%$ of particulates in the composite.

- There is better dispersion of the particulates in the maerix in both verities of composites.

\section{REFERENCES}

[1] Krishnan B P and P K Rohatgi, "Modification of Al$\mathrm{Si}$ alloy melts containing Graphite particle dispersions", Journal of Materials Technology, Vol 11, 1984, pp 41-43.

[2] Andrews Mortensen, J A Cornie and M C Flemings, "Columnar dendritic solidification in a metal matrix composite", Journal of Metallurgical Transactions, Vol 19A, 1988, pp 709-721.

[3] Cirino M, K Fredrich and R B Pipes, "The effect of fibre orientation on abrasive wear behaviour of polymer composite materials", Journal of Wear, Vol 121,1988 pp 127-141.
[4] Rohatgi P K, S Ray and Y Liu, "Tribological properties of metal matrix Graphite composites", International Materials Review, Vol 37, 1992 pp 129-149.

[5] Kim J K, M Kestursatya and P K Rohatgi, "Tribological properties of centrifugally cast Copper alloy Graphite particle composite", Metallurgical and Materials Transactions A, Vol 31A, 2000, pp 1272-1283.

[6] Singh M, A K Jha, S Das and A H Yagneswaran, "Preparation and properties of cast Aluminium alloy-Graphite particle composite", Journal of Material Science, Vol 35, 2000, pp 4421-4426.

[7] Modi O P, "Two body abrasion of a cast Aluminium-Copper (2014Al) alloy-Alumina particle composite influence of heat treatment and abrasion test parameters", Journal of Wear, Vol 248, 2001,pp 100-111.

[8] Das S, D P Mandal and G Dixit, "Correlation of abrasive wear with microstructure and mechanical properties of pressure die cast Aluminium-hard particle composite", Journal of Wear, Vol 253, 2002, pp 176-185.

[9] Sahin Y and M K Acilar, "Properties of SiC particle reinforced Aluminium alloy composite", Journal of Composites, Part A, Vol 34, 2003, pp 709-718.

[10] Pradeep L Menezes, Kishore and Satish V Kailas, "Influence of surface texture on coefficient of friction and transfer layer formation during sliding of pure Magnesium pin on 080 M40 (EN8) steel plate", Journal of Wear, Vol 261, 2006, pp 578-591.

[11] N. Kamp, N. Gao, M.J. Starink \& I. Sinclair, "Influence of grain structure and slip planarity on fatigue crack growth in low alloying artificially aged 2xxx aluminium alloys", International Journal of Fatigue, 29 (2007), 869-878.

[12] K.G. Basavakumar, P.G. Mukunda \& M. Chakraborty, "Impact toughness in $\mathrm{Al}-12 \mathrm{Si}$ and $\mathrm{Al}-$ $12 \mathrm{Si}-3 \mathrm{Cu}$ cast alloys-Part 1: Effect of process variables and microstructure", International Journal of Impact Engineering, 35 (2008), 199-205. 
[13] Wang D, Nia D. R \& Ma Z. Y, "Effect of pre-strain and two-step aging on microstructure and stress corrosion cracking of 7050 alloy”, Material Science Engineering A, 143(2008), 298-303.

[14] Sangshik Kim, James T. Burns \& Richard P. Gangloff, "Fatigue crack formation and growth from localized corrosionin $\mathrm{Al}-\mathrm{Zn}-\mathrm{Mg}-\mathrm{Cu}$ " Engineering Fracture Mechanics, 76 (2009), 651-667.

[15] Mohammad Tajally, Zainul Huda \& H.H. Masjuki, "A comparative analysis of tensile and impacttoughness behavior of cold-worked and annealed 7075 aluminum alloy", International Journal of Impact Engineering, 37 (2010), 425-432

\section{BIOGRAPHIES}

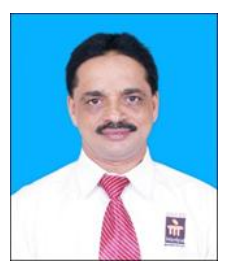

Dr. S. S. Sharma is the First author and corresponding author and holds Bachelor's degree in Industrial \& Production Engineering (Mysore University, India 1987), Master's degree in Materials Engineering (Mangalore University, India 1996) and $\mathrm{PhD}$ degree in Materials Engineering (Manipal University, India 2007). He has 24 years of teaching experience. His area of interest includes Heat Treatment, Deformation of Metals, Material Characterization, Composite Materials. He has published 29 research papers in journals and presented 49 research papers in conferences.www.manipal.edu

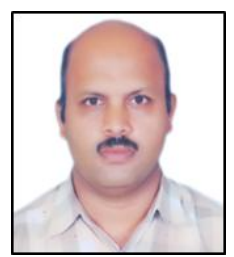

Mr. Gowri shankar M. C is received his Bachelor's Degree in Mechanical Engineering (Mysore University, India 1998) and Master's Degree in Precision and Quality Engg (IIT Kharagpur, India 2006). He has to his credit 7 years of professional experience in industry and more than six years in teaching and research. His area of interest includes Manufacturing, Heat treatment and Metallurgy. He is a faculty member at Manipal Institute of Technology, Manipal and carrying his research in the area of heat treatment of composites from Manipal University. He has published 3 research papers in journals and presented 5 research papers in conferences. www.manipal.edu

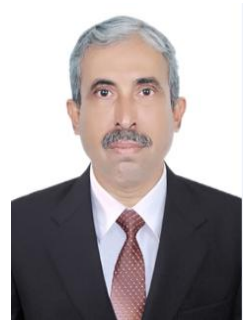

Dr. Achutha Kini holds Bachelor's degree in Mechanical Engineering (Mysore University, India 1985), Master's degree in Engineering Management (Mangalore University, India 1991) and $\mathrm{PhD}$ degree in Corrosion Engineering (Manipal University, India 2012). He has 24 years of teaching experience. His area of interest includes Corrosion Science and Engineering, Composite Materials. He has published 19 research papers in journals and presented 34 research papers in conferences.

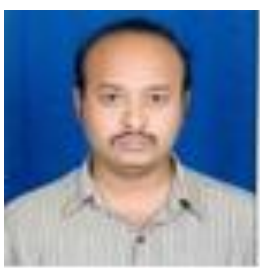

Mr. Shivaprakash Y.M working as Assistant Professor(sr.scale) in the Department of Mechanical and Manufacturing Engineering, MIT, Manipal,Karnataka,India. He did his B.E in Mechanical Engineering from Gulbarga University and M.E in Machine design from Bangalore University. Presently he is pursuing the Ph.D (composite materials) under the supervision of Dr.K.V.Sreenivasa Prasad at SJCE, Mysore, affiliated to VTU, Belgaum. www.manipal.edu

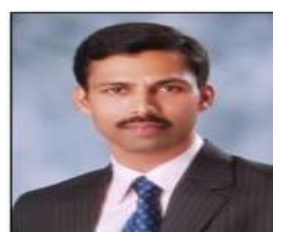

Dr. P. R. Prabhu received his Bachelor's Degree in Mechanical Engineering (Mangalore University, India 2001) and Master's Degree in Engineering Management (MAHE University, India 2003) and $\mathrm{PhD}$ degree in manufacturing (MAHE University, India 2003). He has to his credit 15 months of professional experience in industry and more than nine years in teaching and research. His area of interest includes Manufacturing, Metallurgy, Design of Experiments for Mechanical Engineering Problems. He has published 15 research papers in journals and presented 30 research papers in conferences 